\title{
Diacronie
}

Studi di Storia Contemporanea

$N^{\circ} 28,4 \mid 2016$

La voce del silenzio

\section{Nota introduttiva n. 28 - dicembre 2016}

\section{Alessandro Salvador}

\section{(2) OpenEdition}

\section{Journals}

\section{Edizione digitale}

URL: http://journals.openedition.org/diacronie/4387

DOI: 10.4000/diacronie.4387

ISSN: 2038-0925

\section{Editore}

Association culturelle Diacronie

\section{Notizia bibliografica digitale}

Alessandro Salvador, « Nota introduttiva n. 28 - dicembre 2016 », Diacronie [Online], № 28, 4| 2016

Messo online il 29 décembre 2016, consultato il 24 septembre 2020. URL : http://

journals.openedition.org/diacronie/4387 ; DOI : https://doi.org/10.4000/diacronie.4387

Questo documento è stato generato automaticamente il 24 settembre 2020.

Creative Commons License 
Nota introduttiva n. 28 - dicembre 2016

Alessandro Salvador

La voce del silenzio. Intelligence, spionaggio e conflitto nel XX secolo 
1 Lo spionaggio e l'intelligence militare sono fenomeni di grande rilievo nella storia del $X X$ secolo verso i quali la storiografia ha mostrato un crescente interesse solo in tempi relativamente recenti. Si tratta di argomenti la cui complessità è determinata dalle numerose sfaccettature che il concetto di intelligence può assumere, nonché dalla difficoltà di accesso alle fonti e dalla lentezza e

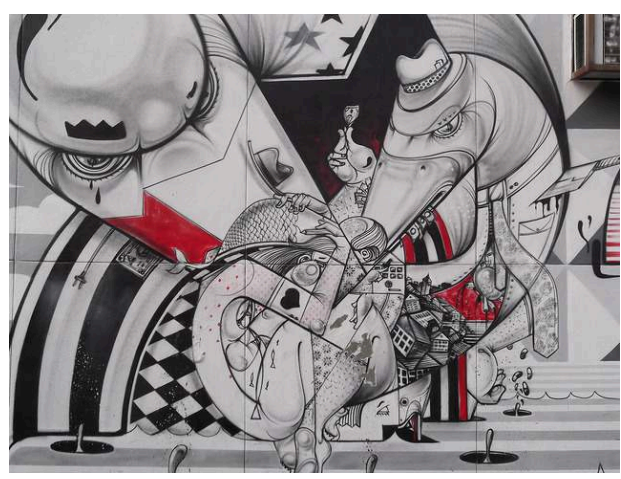
incompletezza con cui queste vengono declassificate. Questo numero monografico di Diacronie vuole offrire dei contributi che possano rendere conto di questa complessità e della varietà dei modi e degli scopi della raccolta di informazioni a scopo militare.

2 Nella prima sezione (I. Spie e soldati: intelligence militare in tempo di guerra) vengono illustrati alcuni aspetti peculiari della raccolta di informazioni in tempo di guerra. Verena Moritz offre un interessante introduzione alla problematica dell'utilizzo dei prigionieri di guerra come fonte di informazioni e alle strategie messe in atto dai servizi segreti austro-ungarici per sfruttare al meglio questa risorsa. Hernán Rodríguez Velasco e Daniel Macías Fernández offrono un quadro delle strutture e delle reti di intelligence militare in contesti specifici: quello della Guerra civile spagnola e della guerra del Rif.

3 La seconda sezione (II. Oltre le linee nemiche: reti di spie e sabotatori), dedicata alle operazioni di intelligence e sabotaggio oltre le linee nemiche, è forse quella in cui la ricerca storiografica si trova a riflettere maggiormente gli stereotipi dell'immaginario collettivo. Ne è un caso esemplare la storia del diplomatico britannico, e spia, Peter Tennant, alias "Karlsson", ricostruita nell'articolo di Victor Lundberg. Il contributo di Nicola Tonietto, invece, offre una visione introduttiva della tematica delle operazioni di intelligence delle forze dell'Asse oltre le linee nemiche durante l'avanzata degli Alleati in Italia. Javier Cervera Gil presenta il caso delle infiltrazioni franchiste nel fronte repubblicano della Guerra civile spagnola.

4 I saggi sullo spionaggio transnazionale, contenuti nella terza sezione (III. I fronti invisibili: lo spionaggio transnazionale), offrono una panoramica ampia non solo delle molte modalità con cui lo spionaggio si può presentare ed esercitare, ma anche dei fenomeni correlati, come la paranoia collettiva e la repressione, che a loro volta possono diventare strumenti bellici. L'articolo di Frank Jacob ci introduce ai servizi segreti ante litteram, le società segrete, e al loro ruolo nei conflitti moderni con l'esempio della guerra russo-giapponese. Manuel Ros Agudo ci offre, invece, una panoramica del problema dello spionaggio in Spagna durante la guerra civile e la seconda guerra mondiale. Gli articoli di Antonio César Moreno Cantano, Pedro Barruso Barés, Emilio Grandío Seoane e José Ramón Rodríguez Lago mostrano, con diverse prospettive, l'importanza delle operazioni di intelligence in paesi neutrali o terzi durante conflitti di grande importanza e in particolare della Spagna come crocevia dello spionaggio durante la Seconda guerra mondiale. Una prospettiva, questa, proposta anche dal contributo di Francisco Javier Juárez Camacho sul ruolo del conglomerato di imprese tedesche SOFINDUS come copertura per operazioni di intelligence in Spagna. Il numero si chiude con due articoli sulla Guerra fredda, il 
periodo che più di ogni altro richiama alla mente la "guerra di spie" e la paura del "nemico interno" della quinta colonna. Federica Addis descrive le attività di spionaggio e controspionaggio della Repubblica Democratica Tedesca volte a supportare, con l'intelligence, il delicato periodo della distensione con l'Occidente. Erzsébet Árvay, invece, illustra i risultati della paranoia da spionaggio con un articolo su due casi celebri, con esiti diversi, di "moderna inquisizione" nei confronti di due grandi personaggi del mondo dello spettacolo americano avvenuti durante il periodo del maccartismo.

5 Riproponiamo poi, in una sezione dedicata (IV.), le Panoramiche di Diacronie per l'anno 2015: una rassegna dei libri che hanno suscitato maggior dibattito in un'area geografica o che sono stati ritenuti degni di una segnalazione da parte dei recensori perché particolarmente importanti nel novero della produzione storiografica dell'anno precedente.

6 Chiude questo numero il consueto spazio dedicato alle recensioni (V.), in quest'occasione particolarmente nutrito.

7 Per tutti gli articoli in spagnolo pubblicati su questo numero la curatela è di Antonio César Moreno Cantano; il coordinamento redazionale è a cura di Alessandro Salvador. Buona lettura,

9 Alessandro Salvador

\section{AUTORE}

\section{ALESSANDRO SALVADOR}

Alessandro Salvador si è laureato in Storia Contemporanea all'Università di Trieste nel 2006 e, nel 2010, ha conseguito il Dottorato di Ricerca in Studi Storici presso l'Università di Trento con una tesi dal titolo "Il partito nazionalosocialista e la destra radicale tedesca nelle fasi finali della Repubblica di Weimar: 1925-1933". Tra le sue pubblicazioni vi sono il volume La guerra in tempo di pace. Gli ex combattenti e la politica nella Repubblica di Weimar (Trento, Università degli Studi di Trento, 2013) e alcuni saggi sul combattentismo e sulla smobilitazione dei soldati ex austroungarici in Italia dopo la Grande guerra. Attualmente è assegnista di ricerca presso l'Università di Trento e collabora col progetto http://www.lagrandeguerrapiu100.it, col progetto europeo CENDARI (Collaborative European Digital ArchivalInfrastructure) e col progetto "World War II Everyday Life Under GermanOccupation" dell'Herder-Institut di Marburg. URL: < http://www.studistorici.com/2015/10/16/alessandro_salvador/ > 